\title{
Alteration of Pectoral Fin Nerves following Ablation of Fin Buds and by Ectopic Fin Buds in the Japanese Medaka Fish
}

\author{
Hitoshi OKAMOTO,1 AND JohN Y. KUWADA*,† \\ ${ }^{*}$ Department of Biology, $\uparrow$ Neuroscience Program, and $¥$ Institute of Gerontology, University of Michigan, Ann Arbor, Michigan 48109
}

Accepted March 26, 1991

\begin{abstract}
The role of the pectoral fin bud for outgrowth by fin axons was assessed by ablation of pectoral fin buds and by transplantation of fin buds to ectopic sites in the embryos of the Japanese medaka fish (Oryzias latipes). Normally nerves from segments 1-4 (S1-4) and less frequently the S5 nerve converged at the base of the fin bud by extending toward the fin bud on the ventral surface of the axial muscles (H. Okamoto and J. Y. Kuwada, 1991, Dev. Biol. 146). Fullowing ablation of the fin bud before motor growth cones have begun to extend laterally, nerves in S1-5 followed a trajectory down the middle of each segment parallel to the borders of the metamerically arranged axial muscles rather than converging. This trajectory was similar to that of more posterior segmental nerves which do not converge toward the fin bud. When fin buds were transplanted to more posterior segments, nerves from S1-5 often changed their trajectories and extended to the base of ectopic buds. Furthermore, motor nerves from segments posterior to $\mathbf{S} 5$, which normally do not innervate the fin bud, also extended to the ectopic fin bud. When faced with both the host and ectopic fin bud, motor nerves extended to either fin bud or branched and extended to both fin buds. These results demonstrate that the early fin bud is necessary for correct outgrowth of fin nerves and suggest that the fin bud normally attracts fin nerves to its base. One possible mechanism for the attraction of motor growth cones by the fin bud is a long distance cue emitted by the fin bud. (c) 1991 Academic Press, Inc.
\end{abstract}

\section{INTRODUCTION}

The role of the limb bud for outgrowth to and formation of a plexus at the base of the limb bud by motor axons has been most extensively examined by a combination of ablation and transplantation studies in amphibians and chicks. Transplantation of limb buds to ectopic sites suggested that axons are attracted to limb buds (Detwiler, 1934, 1936; Hamburger, 1939; Platt, 1956; Hollyday and Hamburger, 1976) and that a long distance cue may be involved in guidance of axons to the limb. Ablation of limb buds, on the other hand, has given results which appear to be either contrary to an attractive influence of limb buds on axons or are inconclusive. For example, in chicks a plexus forms following ablation of the limb bud (Shorey, 1909; Hamburger, 1934, 1958; Oppenheim et al., 1978), but axons within the plexus run uniformly in a posterior direction, including those which normally run anterior (Tosney and Landmesser, 1984). These results are not inconsistent with the finding that limb buds attract segmental nerves. First, the brachial plexus contains the axons which innervate structures at the base of the wing, e.g., shoulder muscles, as well as in the wing proper. Following wing

\footnotetext{
${ }^{1}$ Present address: National Institute for Basic Biology, 38 Nishigonaka, Myodaiji-cho, Okazaki, Aichi 444, Japan.
}

bud ablations usually some shoulder muscles developed. This indicates that the ablations were often incomplete and that some brachial plexus targets were left intact. Second, it is possible that segmental nerves take a default posterior pathway, which was unmasked by limb bud ablations, in the absence of the attraction normally provided by the limb bud.

The pectoral fin and fin motor neurons of the Japanese medaka fish make up a simple system which possesses features that make it attractive for uncovering mechanisms of growth cone guidance (Okamoto and Kuwada, 1991). The pattern of outgrowth by motor axons in wildtype and finless mutant $(p l)$ embryos suggested the hypothesis that fin axons converge and form a plexus at the base of the fin bud because they are attracted by the fin bud. Given the uncertainty associated with past limb bud ablations, we have reexamined this question in two ways. First, we analyzed motor outgrowth following ablation of the pectoral fin bud. The Japanese medaka embryo is well suited for fin bud ablations since the location and orientation of the fin bud with respect to the body trunk make it possible to completely remove it without damaging the substrates motor axons extend upon to get to the base of the fin bud. Second, we analyzed motor outgrowth following transplantation of fin buds to ectopic sites. The pattern of outgrowth by segmental nerves in both cases support 

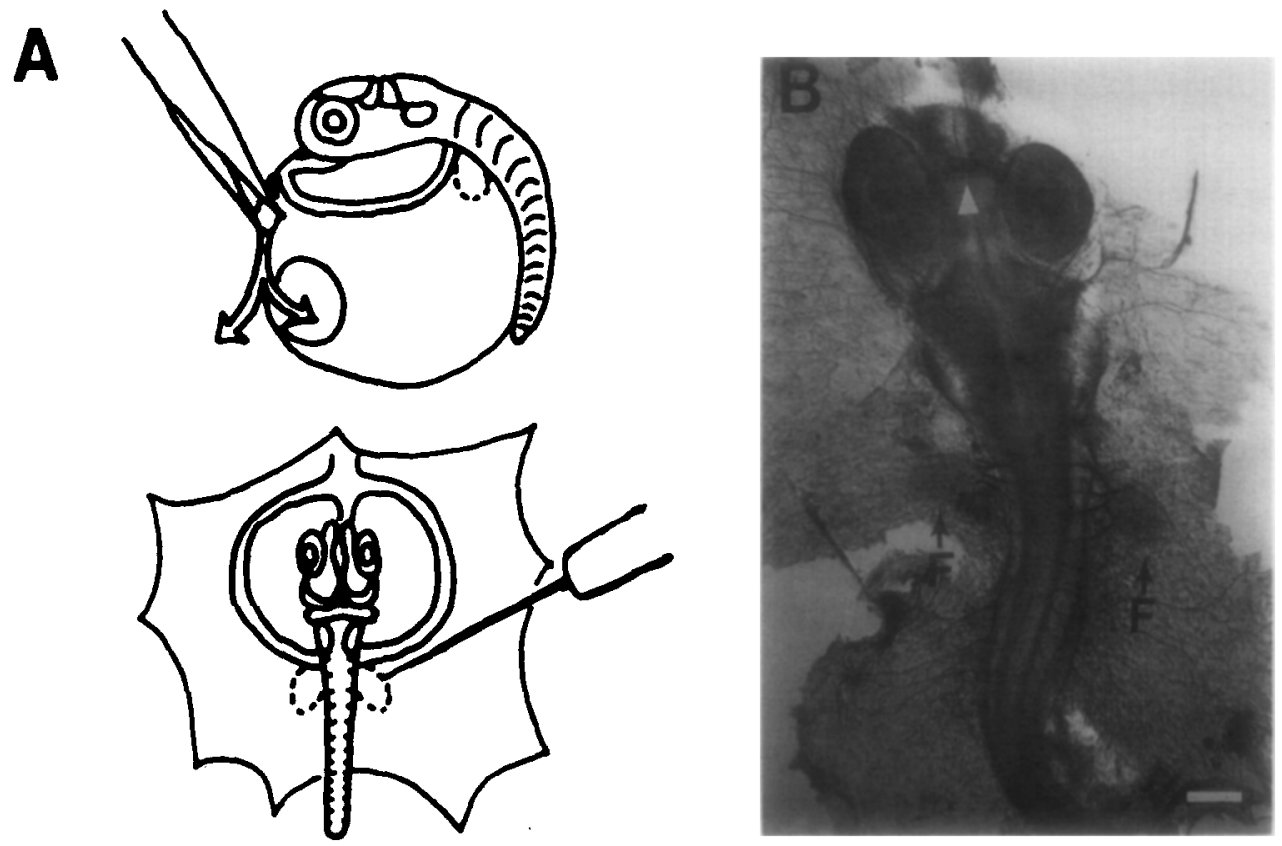

Fig. 1. Embryo cultures of the Medaka fish. (A) Illustration of dissection for the embryo culture procedure (upper) and fin bud ablations (lower). See Materials and Methods for details. (B) Micrograph of an embryo cultured for $36 \mathrm{hr}$. During this time the optic chiasm (arrowhead) formed and the number of spinal segments increased from 19 to 31 . F, pectoral fin bud; scale, $200 \mu \mathrm{m}$.

the hypothesis that they converge at the base of the fin bud because they are attracted by the fin bud.

\section{MATERIALS AND METHODS}

Generation of Embryos and Labeling Peripheral Nerves

Methods for producing, collecting, and maintaining embryos are given in the companion paper (Okamoto and Kuwada, 1991). All methods for labeling segmental nerves are also found in the companion paper.

\section{Ablation of Fin Buds}

Fifty-four-hour embryos (stage 25) still in their chorions were briefly immersed in betadine and washed several times in sterile Yamamoto's saline (Yamamoto, 1976). Embryos were dissected free from their chorions and the yolk sacks torn along the ventral midline with sharpened forceps (Fig. 1). The yolk was gently washed away and the yolk sack was stretched and stuck onto the bottom of plastic culture dishes by pressing the edges of the yolk sack onto the culture dish. Following two changes of sterile Yamamoto's saline, embryos were placed in Earle's balanced salt solution and their fin buds were manipulated. Fin buds develop just posterior to the vitelline vein, but at this stage nascent fin buds were not conspicuous. Nevertheless, excision of the lateral somatopleure just posterior to the vitelline vein, which gives rise to the fin bud, was effective in eliminating the fin bud. Ablations were performed on both sides of embryos since ablation of only one side caused the embryo to bend in the direction of the ablated side and made it difficult to accurately determine the directionality of the fin nerves. Embryos were then cultured in Leibowitz L15 medium containing $10 \mathrm{~m} M$ Hepes buffer (pH 7.4), 15\% fetal bovine serum, $100 \mathrm{units} / \mathrm{ml}$ penicillin, $0.1 \mathrm{mg} / \mathrm{ml}$ streptomycin, and $0.25 \mu \mathrm{g} / \mathrm{ml}$ amphotericin B. Cultures were kept at $26^{\circ} \mathrm{C}$ for 1.5 days and then fixed for immunohistochemistry.

\section{Transplantation of Fin Buds}

Fifty-four-hour embryos were cultured with their yolk sacks stretched on the culture dish as described above. Donor pectoral fin buds and tail buds were dissected from 78 and $54 \mathrm{hr}$ embryos, respectively, with a sharpened tungsten needle. The donor buds were transplanted to sites on one side of 54-hr-host embryos by making a cut at the site and inserting the buds. The contralateral sides served as controls for the experimental sides. In some cases the host fin bud was ablated prior to transplantation of the donor fin bud by excising the somatopleure which gives rise to the fin bud. When this was done at $54 \mathrm{hr}$ there was no regeneration of a new host fin bud. After the transplantations embryos were cultured for 2.5 days at room temperature. 


\section{RESULTS}

\section{Oufgrowth by Fin Axons following Ablation of the Pectoral Fin Bud}

Embryos were grown in embryo culture to assess outgrowth by fin axons in the complete absence of the pectoral fin bud, because it was difficult to completely ablate the fin bud without fatally damaging the embryo. Embryos with their yolk sacks attached to the bottom of culture dishes had their nascent fin buds lateral to the main trunk of the embryo with its base attached to the bottom of the culture dish and the proximal/distal axis of the fin bud parallel with the dorsal/ventral axis of the rest of the embryo (Fig. 1). This geometry allowed for complete ablations of the nascent fin bud including its base without damage to the adjoining tissues such as the somites. Embryos were cultured starting at $54 \mathrm{hr}$. (stage 25) and left in culture for 1.5 days (see Materials and Methods). In vivo at $54 \mathrm{hr}$ the fin bud is not conspicuous and motor growth cones from S1-5 are normally extending ventrally between the notochord and the medial surface of the axial muscles (Okamoto and Kuwada, 1991). They reach the ventral surface of the axial muscles and begin to extend laterally towards the fin bud 2-3 hr later.

Control embryos without any manipulations $(n=10)$ appeared to develop normally (Fig. 1B). For example, retinal axons formed a normal optic chiasm, the number of segments increased from 19 to 31 as it does in vivo, and embryos even became motile. However, the fin bud developed at a slower rate than it would have in vivo. The fin bud increased in size and developed an apical ectodermal ridge (AER), but did not increase in size as much as it would have during 1.5 days in vivo and the fin mesenchyme did not begin to differentiate into muscle and cartilage. Judging from the state of development of the fin bud, cultured embryos underwent $16 \mathrm{hr}$ of development ( $70 \mathrm{hr}$, late stage 27 ; Fig. 2B). Despite the slower rate of development segmental nerves extended normally on the ventral surface of the axial muscles (Figs. $1 \mathrm{~B}$ and $2 \mathrm{~A}$ ). Nerves from S1-1 converged to form a plexus at the base of the fin bud and nerves from more posterior segments extended laterally and caudally down the middle of the ventral surface of the axial muscles. The S5 nerve projected no anterior branch, but in vivo this branch does not appear until approximately 84 $\mathrm{hr}$. The $\mathrm{S} 1$ and $\mathrm{S} 4$ nerves branched but often the anterior branch of $\mathrm{S} 1$ and the posterior branch of $\mathrm{S} 4$ were thinner than they are in vivo.

Following embryo culture and complete ablation of nascent fin buds at $54 \mathrm{hr}$, the fin bud did not regenerate (Fig. 2D) and the nerves from S1-4 did not converge and did not form a plexus (Fig. 2C). Instead, the nerves had trajectories which resembled those of the more poste- rior segments in 36 of 38 cases; nerves ran laterally along the middle of the ventral surface of the axial muscles. In most of these cases the S1 and S4 nerves did not branch normally although occasionally nerves did extend parallel branches. In the other 2 cases convergence was abnormal: nerves from $\mathrm{S} 1$ and $\mathrm{S} 2$ converged, but $\mathrm{S} 3$ and $\mathrm{S} 4$ ran laterally; nerves from $\mathrm{S} 1$ and $\mathrm{S} 2$ converged together and S3 and S4 converged together, but all four nerves did not converge. Additionally, ablation of the fin bud in $p l$ embryos, which have defective fin bud development (Okamoto and Kuwada, 1991), had the same effect on segmental nerves as removal of fin buds in wildtype. Again the nerves from S1-4 ran laterally on along the ventral surface of the axial muscles in parallel to the muscle borders and did not converge $(n=3$; data not shown).

It is not likely that the ablation procedure inadvertently disturbed or damaged the trunk proximal to the fin bud or at the junction between the fin bud and the body trunk for two reasons. First, in 7 cases a fin bud was present despite the ablation procedure. In these cases nerves from S1-4 converged normally (data not shown). We presume these cases represent incomplete ablations, since in 38 of 45 cases no fin buds were present, but cannot strictly rule out that in some cases regeneration of the fin bud can occur. Second, in sham experiments in which the nascent fin bud was cut off from the body trunk but left in place, nerves from $\mathrm{S} 1-4$ converged to form a plexus at the base of the fin bud in 4 of 5 embryos (Fig. 2E).

\section{Outgrowth of Segmental Nerves following Ablation of Host Fin Bud and Transplantation of Donor Fin Bud}

The larger fin buds of 78-hr (late stage 28) embryos were used as donor fin buds and transplanted to $54 \mathrm{hr}$ host embryos since the fin buds of earlier embryos proved to be too small and difficult to transplant reliably (see Materials and Methods). Furthermorc, fin buds from 78-hr donors probably influence segmental nerves since convergence of all the fin nerves is not completed until later in development (Okamoto and $\mathrm{Ku}$ wada, 1991). The fin bud from one side of the host embryos were ablated just prior to transplantation of donor fin buds.

The fin bud is normally centered on the $\mathrm{S} 1 / \mathrm{S} 2$ border. Following ablation of host fin buds and transplantation of donor fin buds to more posterior positions, segmental nerves near the ectopic fin bud converged to the base of the ectopic bud $(n=7)$. Three aspects of these results are especially noteworthy. First, segmental nerves from S1-5, which normally converge toward the fin bud, did so toward orthotopically transplanted donor fin buds (Figs. 3A and 3B). Second, both nerves from segments 

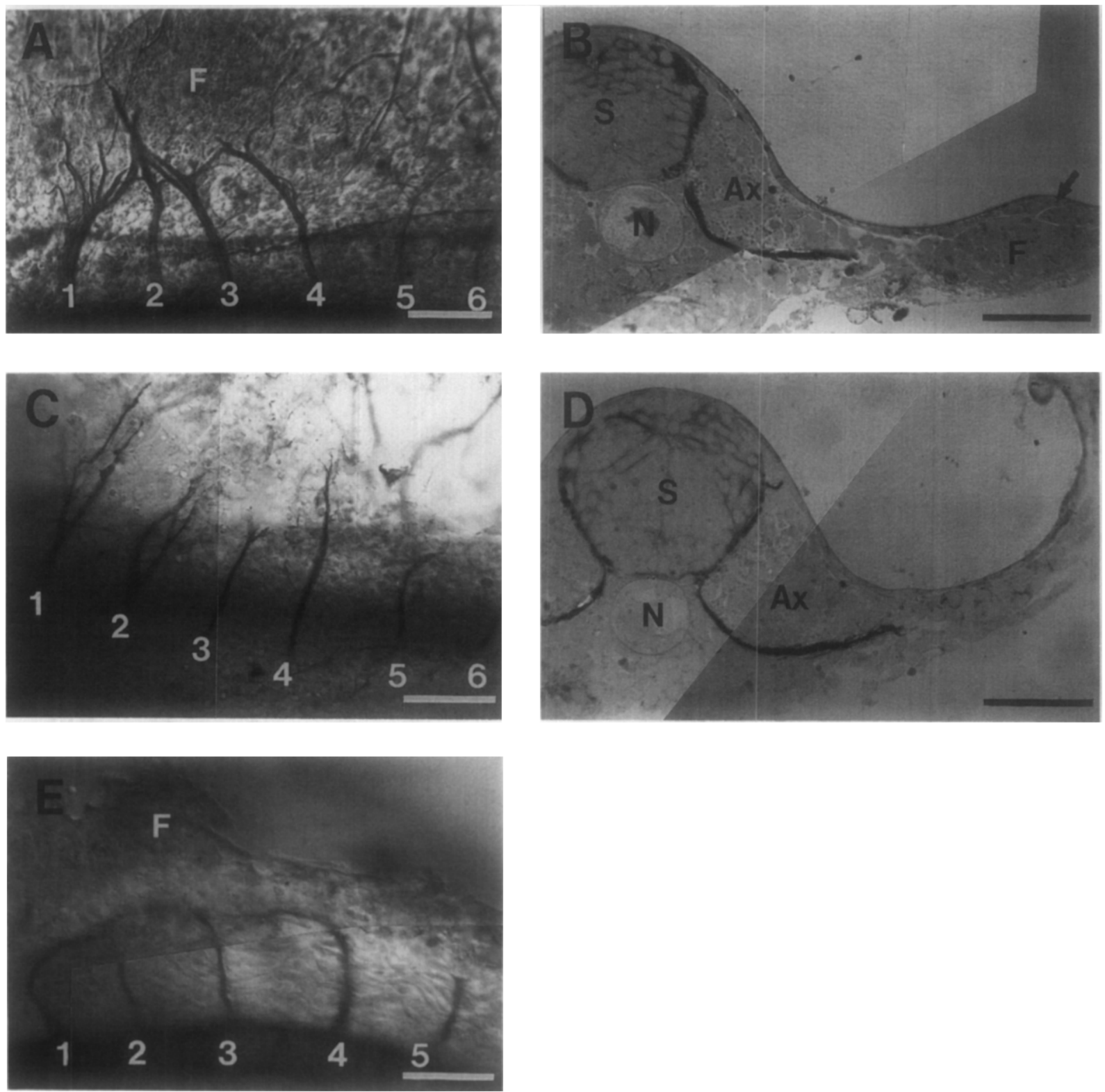

FIG. 2. Outgrowth of segmental nerves in eultured nonoperated embryos (A, B), fin bud ablated embryos (C, D), and sham operated embryos (E). (A, C, and E) Ventral views of embryos with segmental nerves labeled by the acetylated tubulin antibody. In the nonoperated and sham operated controls nerves from S1-4 converged to the fin bud, F, but ran straight laterally following ablation of fin buds. Numbers denote the segments from which the nerves originate; anterior, left; lateral, up. (B) and (D) are cross sections showing acetylated tubulin labeled nerves run ventrally and laterally along their normal substrates in the presence and absence of the fin bud. In the experimental embryos there was no fin bud regeneration and the wound healed. S, spinal cord; N, notochord; Ax, axial muscles; arrow, cleft in the AER. Scale for (A-E), $50 \mu \mathrm{m}$.

which normally converge to the fin bud and those from segments which normally do not can converge to the base of the ectopic fin bud. For example, the S6 nerve never converges to the fin bud normally but does so towards a larger donor bud lateral to $\mathrm{S} 1-4$ (Figs. $3 \mathrm{C}$ and 3D). Third, segmental nerves change their trajectories in order to reach the fin bud (Figs. 3E and 3F). The S2-4 nerves normally extend along a diagonal pathway both anteriorly and laterally to reach the fin bud, but extend posteriorly and laterally to reach a more posterior fin bud. 'The nonfin $\$ 6$ nerve branches laterally with one branch running anteriorly and the main trunk of the S5 nerve running anteriorly and laterally towards the ectopic fin bud. Normally the S6 nerve runs laterally and posteriorly away from the fin bud and does not branch, while the main trunk of the S5 nerve also runs posteriorly and laterally. These results suggest that fin buds can attract segmental nerves to extend to them. 


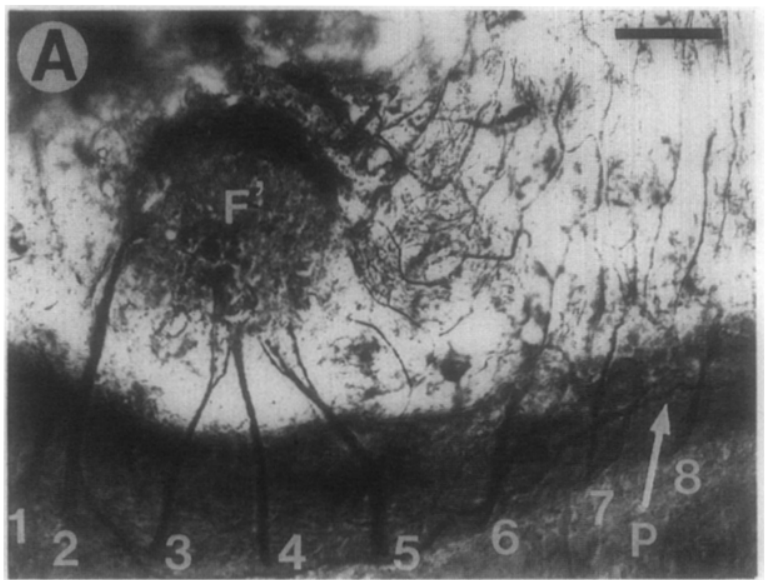

B
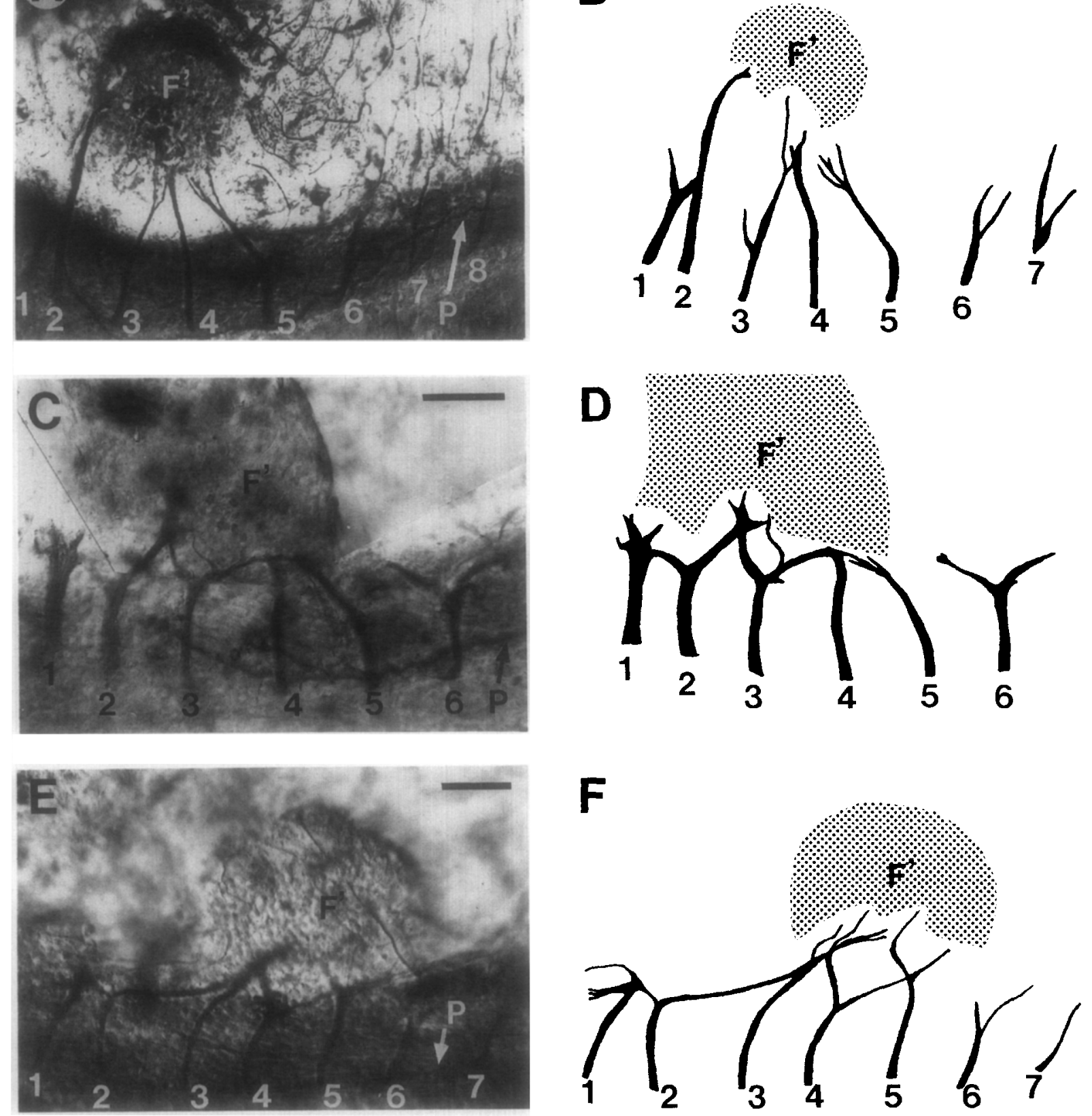

FIG. 3. Segmental nerves converge to the donor fin bud, $F^{\prime}$, following ablation of the host fin bud and transplantation of a donor fin bud. (B, D, and F) Camera lucida drawings of segmental nerves shown in (A, C, and E) respectively. The cilia in the pronephros, $P$, are also labeled with the acetylated tubulin antibody. (A, B) Donor fin bud was transplanted so that it was centered between S3 and S4 and was more lateral than normal. (C, D) Donor fin bud was transplanted immediately lateral to S1-4. Note that the S6 nerve extends an anterior branch toward the donor fin bud. (E, F) Donor tin bud was transplanted just lateral to S3-5. Note that the S2 and S3 nerves extend posteriorly and that the S4 and S5 nerves extend laterally rather than anteriorly. The nerve running across the donor bud are axons of Rohon-Beard neurons in the spinal cord. Scale for (A, C, and E), $50 \mu \mathrm{m}$.

Fig. 4. Segmental nerves extend toward both the host $F$ and donor $F^{\prime}$ fin buds but not toward a donor tail, T, bud. (B, D, F, and H) Camera lucida drawings of (A, C, E, and G). (A, B) Donor fin bud was transplanted to a site anterior and lateral to the host bud. (C, D) Donor fin bud was transplanted to a site lateral and posterior to the host fin bud. The S4 nerve and branches of the S3 nerve have extended towards the donor bud. (E, F) Donor fin bud was transplanted directly posterior to the host bud. Nerves from S5-7 formed a plexus at the base of the donor bud. (G, H) Donor tail bud did not change the trajectories of segmental nerves. The cilia in the pronephros (P) are also labeled. Scale for all panels, $50 \mu \mathrm{m}$. 

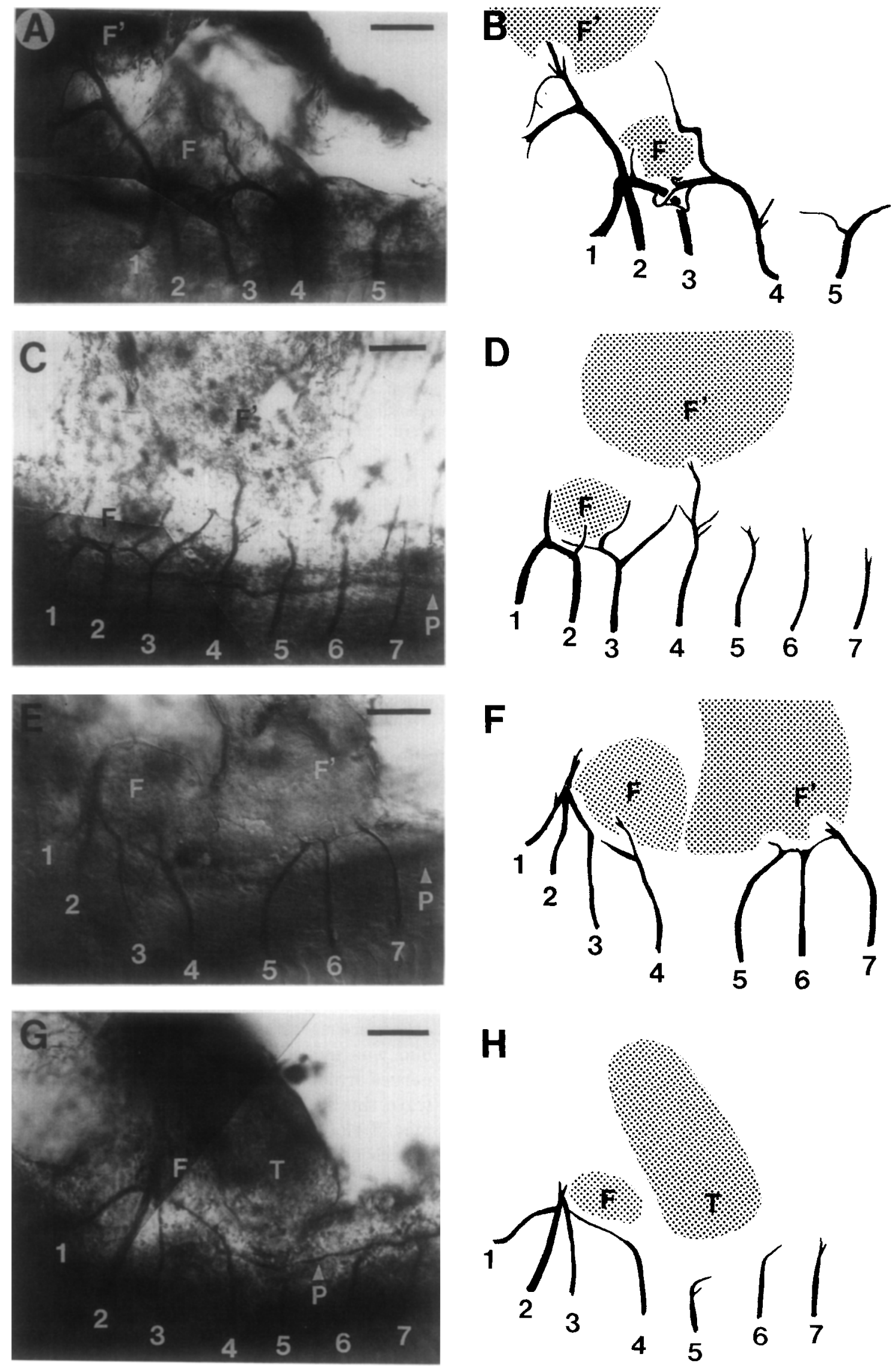

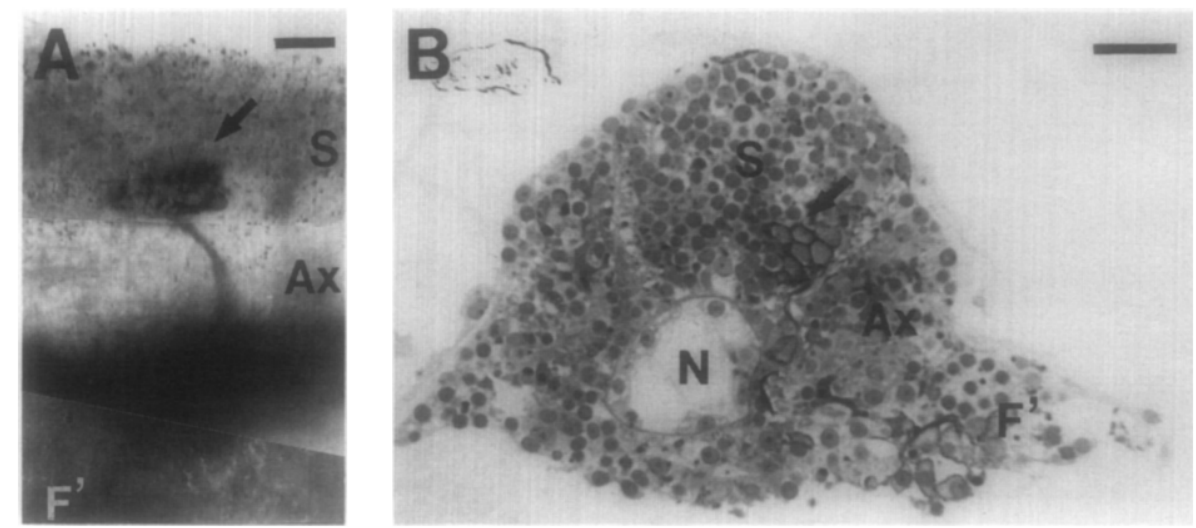

FIG. 5. Putative motor neurons from segments which normally do not innervate the fin bud, but which extended to ectopic fin buds. Motor neurons were back-labeled following application of diI to the base of the donor fin bud, $\mathrm{F}^{\prime}$, followed by photooxidation. (A) Side view of wholemounted embryo showing the labeled cells (arrow) in the ventral portion of S7 of the spinal cord (S) with their peripheral axons. Axial muscles, Ax; anterior, left; dorsal, up. (B) Cross section of another embryo showing labeled ventrolateral neurons (arrow) in S7. The position of these neurons corresponds to the position of motor neurons in the spinal cord. N, notochord; scale for $(A, B), 20 \mu \mathrm{m}$.

\section{Outgrowth of Segmental Nerves following Transplartation of Fin Buds wilhout Ablation of Host Fin Buds}

The trajectories of segmental nerves following the addition of an ectopic fin bud to host embryos $(n=28)$ also suggested that fin buds attract segmental nerves. First, nerves from segments which normally do not converge to the fin bud now often converged toward an ectopic fin bud (Fig. 4). DiI back-labeling from the ectopic fin bud showed that these nerves contained the axons of neurons located in the ventral spinal cord of the segment corresponding to the back-labeled nerve $(n=5$; Fig. 5). The location of these neurons and trajectories of their axons indicate that they are likely to be motor neurons. Second, segmental nerves changed their trajectories to reach ectopic fin buds (Fig. 4). Again, both nerves from segments which normally converge toward the fin bud and segments which normally do not can change their trajectories to reach an ectopic fin bud. Segmental nerves in between the host and donor fin buds either extended to the host fin bud, to the donor fin bud, or branched and extended branches to both (Figs. $4 \mathrm{C}$ and $4 \mathrm{D}$ ). In four cases where the donor fin bud was transplanted to a site at the same segmental level as the host fin bud but lateral to the host bud, nerves from S1-5 converged at the base of the host fin bud but aberrant nerves extended from these nerves towards the donor fin bud (not shown). In contrast transplants of tail buds from 54-hr (stage 25) embryos did not disturb the normal outgrowth patterns of segmental nerves ( $n=6$; Figs. $4 \mathrm{G}$ and $4 \mathrm{H}$ ).

The systematic change in trajectories of segmental nerves as the location of the donor fin bud changed along the anterior/posterior axis can be appreciated in
Fig. 6. As the donor fin bud was moved posteriorly nerves from more posterior segments extended toward the donor fin bud. To estimate the distance the donor fin bud was able to attract segmental nerves, nerves from segments posterior to the donor bud were examined since segmental nerves anterior to the donor bud would potentially be influenced by both host and donor buds. By comparing the ratios of nerves which extended anteriorly toward the donor fin bud from segments successively posterior to the donor bud, it was apparent that the two closest segments were dramatically influenced with the closest nerve more so than the next nerve (Fig. 6). The nerves from segments in between the host and donor fin buds appeared to be influenced by both fin buds. This is apparent when one compares the percentage of nerves or nerve branches from S2 and S3 which extended posteriorly toward the donor fin bud when the donor fin bud was transplanted to different segmental levels. In 4 cases nerves branched and extended to both host and donor fin buds. The percentage of both the S2 and S3 nerves or nerve branches that extended posteriorly increased steadily as the position of the donor fin bud was moved anteriorly (Table 1). Conversely, the nerves or nerve branches which extended anteriorly toward the host fin bud decreased as the donor bud was moved closer to segments S2 and S3. The S2 and S3 nerves normally always extend anteriorly toward the fin bud.

\section{DISCUSSION}

\section{Attraction of Motor Axons by Limb Buds}

The pattern of outgrowth by spinal motor growth cones in wildtype and $p l$ embryos (Okamoto and $\mathrm{Ku}$ wada, 1991), and the trajectories of segmental nerves 


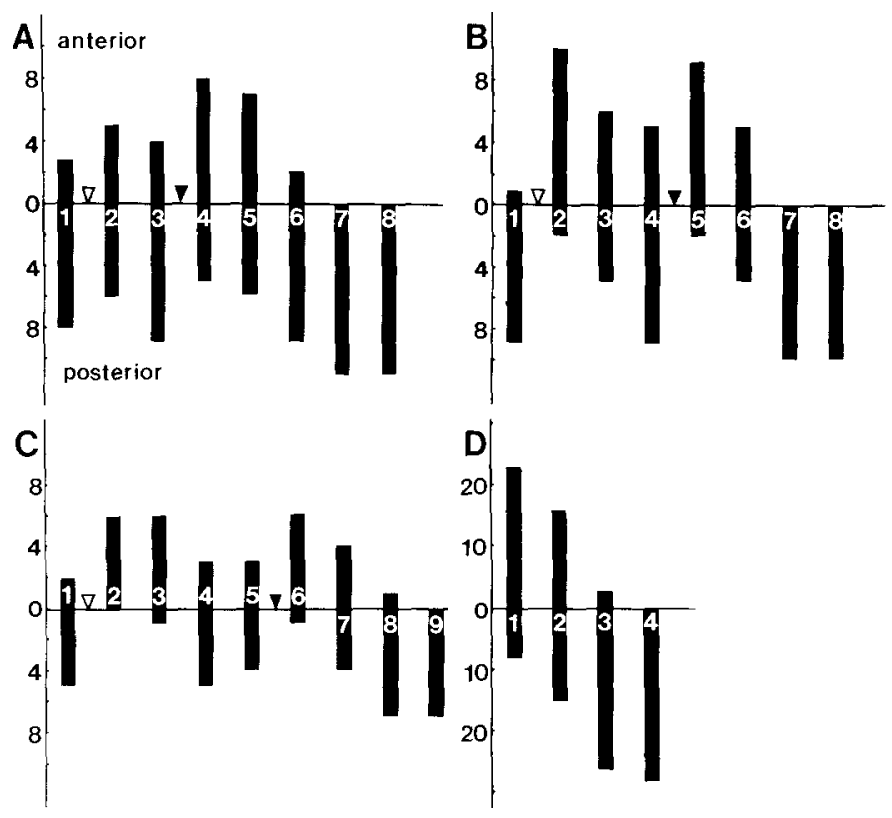

FIr. 6. Directional changes in the trajectories of segmental nerves or nerve branches in response to an ectopic fin bud. In some cases the numbers are larger than the number of nerves (e.g., S3 in A) since some nerves branched. (A) Number of segmental nerves (segment of origin denoted by the numerals) which projected anteriorly or posteriorly when the donor fin bud was centered at the S3/S4 border. The number of nerves projecting anteriorly and posteriorly is designated by a bar above and below the abscissa, respectively. Location of the host fin bud, open triangle; location of the donor fin bud, closed trian gle. (B) Same as (A) except the donor bud was centered at the S4/S5 border. (C) Same as (A) except the donor bud was centered at the S5/S6 border. (D) Compilation of the number of nerves projecting anteriorly and posteriorly taken from $(\mathrm{A}-\mathrm{C})$ which were $1,2,3$, and 4 segments posterior to the center of the site of the donor fin bud.

following fin bud ablations and to ectopic fin buds all suggest that the fin bud normally exerts a long-distance attractive influence on motor growth cones. Following fin bud ablations, the nerves from $\mathrm{S} 1-5$ extended laterally within their own segments and did not converge toward the fin bud much like the nerves from more posterior segments. These results establish that the fin bud is necessary for normal outgrowth and that it is unlikcly that local cues associated with their normal pathway are sufficient to guide axons to the base of the fin bud. In $p l$ embryos the pectoral fin bud begins to develop but soon arrests, and the pattern of outgrowth by segmental nerves was consistent with a diminished capacity of the fin bud to attract growth cones and/or the growth cones to respond to the fin bud. The outgrowth patterns in fin-bud-ablated and $p l$ embryos further suggest the existence of a default pathway in S1-5 perhaps defined by local cues, which is similar to the pathway normally taken by nerves from more posterior segments. Presumably the attraction of nerves from S1-5 by the fin bud overcomes the local default pathway cues in these seg- ments of normal embryos but does not in S4-5 of pl embryos.

The outgrowth pattern of nerves following transplantation of limb buds to ectopic sites provides more compelling evidence for attraction of axons by limb buds. The trajectory of spinal motor axons to ectopic fin buds in the fish embryo is similar to the trajectory of segmental nerves to ectopic limb buds in amphibian (Detwiler, 1934, 1936; Piatt, 1956) and chick (Hamburger, 1939; Hollyday and Hamburger, 1976) embryos in two important ways. First, spinal nerves from segments which normally innervate the limb and segments which do not innervate the limb both extended to ectopic limb buds. Second, segmental nerves changed their trajectories appropriately to reach ectopic limb buds. In another experiment an obstruction was placed along the pathways motor axons normally follow to reach the limb bud (Hamburger, 1929). Despite the presence of a mica plate between the spinal cord and the limb bud, segmental nerves reached the limb bud by running around the obstruction. These findings indicate that attraction of growth cones by limb buds may be a mechanism common to a variety of animals.

Since ectopic fin buds can attract nerves from more posterior segments, these nerves are presumably too far from the fin bud to be attracted by it in normal embryos. Instead they follow the local default pathway cues. The distance over which motor growth cones are attracted by the fin bud in the Japanese medaka can be roughly estimated from the pattern of outgrowth in normal embryos and following fin bud transplantation to be two to three segments. In chicks a similar decline of attraction of segmental nerves with distance from the ectopic limb bud was observed (Hamburger, 1939).

\section{Nerve Outgrowth following Experimental Manipulation of the Limb Bud}

The results of previous limb bud ablalion studies, in contrast to our experiments, either appear to contradict

TABLE 1

Percentage of Segmental Nerves or Nerve Branches which EXTENDED POSTERIorly toward a Donor Fin Bud Transplanted to a Site Posterior to the Host Fin Bud, Which Was Left Intact and CenTERED AT THE S1/S2 Border

\begin{tabular}{lccc}
\hline & \multicolumn{3}{c}{ Site donor fin bud was centered } \\
\cline { 2 - 4 } Nerve & $\begin{array}{c}\text { S3/S4 } \\
(n=11)\end{array}$ & $\begin{array}{c}\text { S4/S5 } \\
(n=10)\end{array}$ & $\begin{array}{c}\mathrm{S} 5 / \mathrm{S} 6 \\
(n=7)\end{array}$ \\
\hline S2 $(\%)$ & 58 & 17 & 0 \\
S3 $(\%)$ & 71 & 45 & 14 \\
\hline
\end{tabular}


the conclusion drawn from transplantation experiments or are inconclusive. In chicks, motor axons extend laterally and then uniformly in a posterior direction to form an abnormal plexus (Tosney and Landmesser, 1984). This result, however, is not inconsistent with attraction of motor axons by the limb bud. In the chick, motor axons may have a tendency to extend posteriorly once they have extended laterally which is normally overridden by their attraction to the limb bud. In fact, the segmental nerves in segments posterior to $\mathrm{S} 4$ in the Japanese medaka embryo extend in a similar pattern. In amphibians a seemingly normal plexus forms in the position corresponding to the base of the limb bud (Shorey, 1909; Hamburger, 1934). However, since the limb regenerates in the species used and despite repeated ablations, it is uncertain and perhaps unlikely that the segmental nerves were never exposed to a regenerating limb bud. In this regard we observed no regeneration of the fin bud in our experiments. Lack of regeneration by fin buds was also observed following early fin bud ablations in another teleost (Nicholas and Oppenheimer, 1942).

The results of previous limb bud ablations may in part be due to the possibility that the ablations were incomplete or damaged adjacent trunk tissue in addition to removing the limb bud. It may be very difficult to completely ablate the limb bud including its base without unduly disturbing the substrates leading to the limb bud in chicks and amphibians since the base of the limb buds in these species directly abuts the trunk of the embryo. Fin bud ablations in the Japanese medaka embryos, however, are likely to be complete because of the unusual relationship of the fin bud to the rest of the embryo (Okamoto and Kuwada, 1991) combined with our culturing technique. These advantages may account for the fact that our ablation results are more obviously consistent with the results from limb (Detwiler, 1934; Hamburger, 1939) and fin bud transplantations.

\section{The Nature of the Attraction of Motor Nerves by Limb Buds}

Limb buds can attract nerves from inappropriate sources in a variety of animals. In some cases limbs are functionally innervated by motor neurons from inappropriate segments (O'Brien and Oppenheim, 1990), but these connections are apparently lost due to the eventual death of the inappropriate motor neurons and the regression of the limb (O'Brien et al., 1990). Furthermore, in amphibians other tissues such as eye rudiments can also attract spinal nerves (Detwiler, 1936). But not any tissue can serve as the source of the attrac- tant since the tail bud failed to attract segmental nerves in both amphibians (Detwiler, 1936) and fish.

The finding that motor axons from inappropriate segments can be attracted by the fin bud raises two questions which are not resolved by our experiments: (1) What is the identity of the motor neurons from inappropriate segments which are attracted to the fin bud? (2) How and by what motor neurons are the axial and fin muscles innervated in those segments which normally innervate the fin? These questions deal with the issues of when and how axial and fin motor neurons become specified and whether all motor neurons are attracted by the fin bud.

The large size of the early motor growth cones and the early branching of the segmental nerves are consistent with the possibility that growth cones may be extending along a gradient of a cue emanating from the fin bud (Okamoto and Kuwada, 1991). Numerous in vitro experiments have demonstrated that growth cones can be directed to a distant target by a long distance cue (Heffner et al., 1990; Lumsden and Davies, 1983, 1986; McCaig, 1986; Tessier-Lavgne et al., 1988) and limb buds can apparently affect outgrowth of neurites from spinal cord explants (Pollack and Liebig, 1977). Suitable in vitro experiments with fish embryos similar to those performed on other systems should demonstrate whether spinal growth cones are attracted by a fin-bud-derived diffusible cue. Our in vivo experiments suggest that the normal outgrowth pattern of segmental nerves results from a combination of local substrate cues and a fin-bud-derived long distance cue which falls off with distance. The long distance cue overcomes the local cues in segments close to the fin bud but is too weak to affect outgrow th in more distant segments. In these segments the local substrate cues determine the pathway followed by motor growth cones.

We thank H. Tomita, A. Shima, and K. Naruse for fish stocks; A. Shima and K. Naruse for advice on the selection of embryo culture medium; G. Piperno for the acetylated tubulin antibody; P. Knafl for technical assistance; D. Bay for photography; and R. R. Bernhardt and $\mathrm{K}$. W. Tosney for reading the manuscript. This work was supported by National Institute of Health Grant NS24848 and March of Dimes Grant 5-687. J.Y.K. is a Basil O'Connor Scholar of the March of Dimes and H.O. is a postdoctoral fellow of the Toyobo Biotechnology Foundation.

\section{REFERENCES}

DETWILER, S. R. (1934). An experimental study of spinal nerve segmentation in Amblystoma with references to the plurisegmental contribution to the brachial plexus. J. Exp. Zool. 67, 395-441.

DETwILER, S. R. (1936). "Neuroembryology." Haffner, New York.

Hamburger, V. (1929). Experimentelle Beiträge zur Entwicklungsphysiologie der Nervenbahnen in der Froshextremität. Wilhelm Roux Arch. 119, 47-99. 
Hamburger, V. (1934). The effects of wing bud extirpation on the development of the central nervous system in chick embryos. $J$. Exp. Zool 68, 449-494.

Hamburger, V. (1939). The development and innervation of transplanted limb primordia of chick embryos. J. Exp. Zool. 80, 147-189.

HAMBURGER, V. (1958). Regression versus peripheral control of differentiation in motor hypoplasia. Am. J. Anat. 102, 365-409.

HeFFner, D. C., LuMSDEN, A. G. S., and O'LeARY, D. D. M. (1990). Target control of collateral extension and directional axon growth in the mammalian brain. Science 247, 217-220.

Hollyday, M., and HAMBURGER, V. (1976). Reduction of the naturally occurring motor neuron loss by enlargement of the periphery. $J$. Comp. Neurol. 170, 311-320.

Lumsden, A. G. S., and DAvies, A. M. (1983). Earliest sensory nerve fibres are guided to peripheral targets by attractants other than nerve growth factor. Nature 306, 786-788.

Lumsden, A. G. S., and Davies, A. M. (1986). Chemotropic effect of specific target epithelium in the developing mammalian nervous system. Nature $323,538-539$.

MCCAIG, C. D. (1986). Myoblasts and myoblast-conditioned medium attract the earliest spinal neurites from frog embryos. J. Physiol. $375,39-54$.

NiCHOLAS, J. S., and OPPENHEIMER, J. M. (1942). Regulation and reconstitution in fundulus. J. Exp. Zool. 90, 127-157.

O'BRIEN, M. K., and OPPENHEIM, R. W. (1990). Development and survival of thoracic motoneurons and hindlimb musculature following transplantation of the thoracic neural tube to the lumbar region in the chick embryo: Anatomical aspects. $J$. Neurobiol. 21, 313-340.
O'BRIEN, M. K., LANDMESSER, L., and OPPENHEIM, R. W. (1990). Development and survival of thoracic motoneurons and hindlimb musculature following transplantation of the thoracic neural tube to the lumbar region in the chick embryo: Functional aspects. J. Neurobiol. $21,341-355$.

OKaмото, H., and Kuwada, J. Y. (1991). Outgrowth by fin motor axons in wildtype and a finless mutant in the Japanese Medaka fish. Dev. Biol. 146,

OPpenheim, R. W., ChU-WANG, I.-W., and Maderdrut, J. L. (1978). Cell death of motorneurons in the chick embryo spinal cord. The differentiation of motorneurons prior to their induced degeneration following limb bud removal. J. Comp. Neurol. 177, 89-111.

PIATT, J. (1956). Studies on the problem of nerve pattern. I. Transplantation of the forelimb primordium to ectopic sites in A mblystoma. $J$. Exp. Zool. 131, 173-202.

Pollack, E. D., and LiEBig, V. (1977). Differentiating limb tissue affects neurite growth in spinal cord culture. Science 197, 899-900.

SHOREY, M. L. (1909). The effect of the destruction of peripheral areas on the differentiation of the neuroblasts. J. Exp. Zool. 7, 25-63.

Tessier-Lavigne, M., Placzek, M., Lumsden, A. G. S., Dodd, J., and JESSEL, T. M. (1988). Chemotropic guidance of developing axons in the mammalian central nervous system. Nature 336, 775-778.

Tosney, K. W., and LANDmesser, L. T. (1984). Pattern and specificity of axonal outgrowth following varying degrees of chick limb bud ablation. J. Neurosci. 4, 2518-2529.

YAмamoto, T. (1976). Series of stock culture in biological field. In "Medaka (Killifish), Riology and Strains." Keigaku Publishing, Co., Tokyo, Japan. 